\title{
FORMACIÓN FILOSÓFICA, LÓGICA Y EPISTEMOLÓGICA DEL INVESTIGADOR
}

\author{
Philosophical, logical and epistemological training of the researcher \\ Cuba-Esquivel, Amadeo 1, : \\ 1 Departamento de ciencias y humaninades, Universidad Andina del Cusco \\ a Doctor en filosofía \\ ¡ acubae@uandina.edu.pe
}

Citar como: Cuba-Esquivel, A. (2018). Formación filosófica, lógica y epistemológica del investigador. Rev Yachay, 7(1), 464-471.

Recibido: 16-09-2018; Aceptado 07-12-2018

\section{Introducción}

El contexto de la globalización y de la difusión vertiginosa de las tecnologías de información y comunicación (las TIC), induce a las instituciones de educación superior a priorizar la investigación con la aspiración de estar a la par con la internacionalización de los avances de la innovación científica y tecnológica. Para llevar a cabo estas expectativas científicas innovadoras, las instituciones universitarias requieren, más que nunca, los recursos humanos con perfil pertinente en el rubro de investigación y producción científica. Sin embargo, cabe preguntarse: ¿las universidades cuentan con investigadores académicos con perfil pertinente?, todo esto para garantizar con sostenibilidad la generación de conocimientos y solución de problemas tecnológicos causados por los cambios continuos en esta nuestra sociedad de la información. La respuesta no es tan alentadora.

Actualmente, los investigadores en ciencias, tecnología e innovación (CTI) en el Perú son muy escasos, tanto en cantidad como en calidad; porque no se ha logrado formar una "masa crítica" de investigadores con aptitud adecuada ni en generación ni en gestión de las CTI, que sean capaces de crear, transferir, adaptar, y adecuar los conocimientos y la tecnología a las necesidades sociales, políticas y económicas y culturales del país (SINACYT - CONCYTEC, 2008).

Las causas de esta situación son múltiples: el escaso apoyo político; poca inversión económica para la investigación en el Perú, el enfoque adoptado por el sistema educativo peruano basado sobre las competencias y acogido en todos los niveles — desde inicial hasta superior- y que no favorece la formación para la investigación.

Según la (Comisión Consultiva para la Ciencia, 2012):

La investigación en el Perú no tiene nivel ni relevancia, peso específico, ni respaldo político que sí tiene en otros países de desarrollo similar. Se encuentra lejos de los países desarrollados y emergentes. Por ejemplo, la inversión en CTI específicamente en investigación y desarrollo (I y D), llega escasamente al 0,1\% del PBI, Una de las más bajas del mundo. 


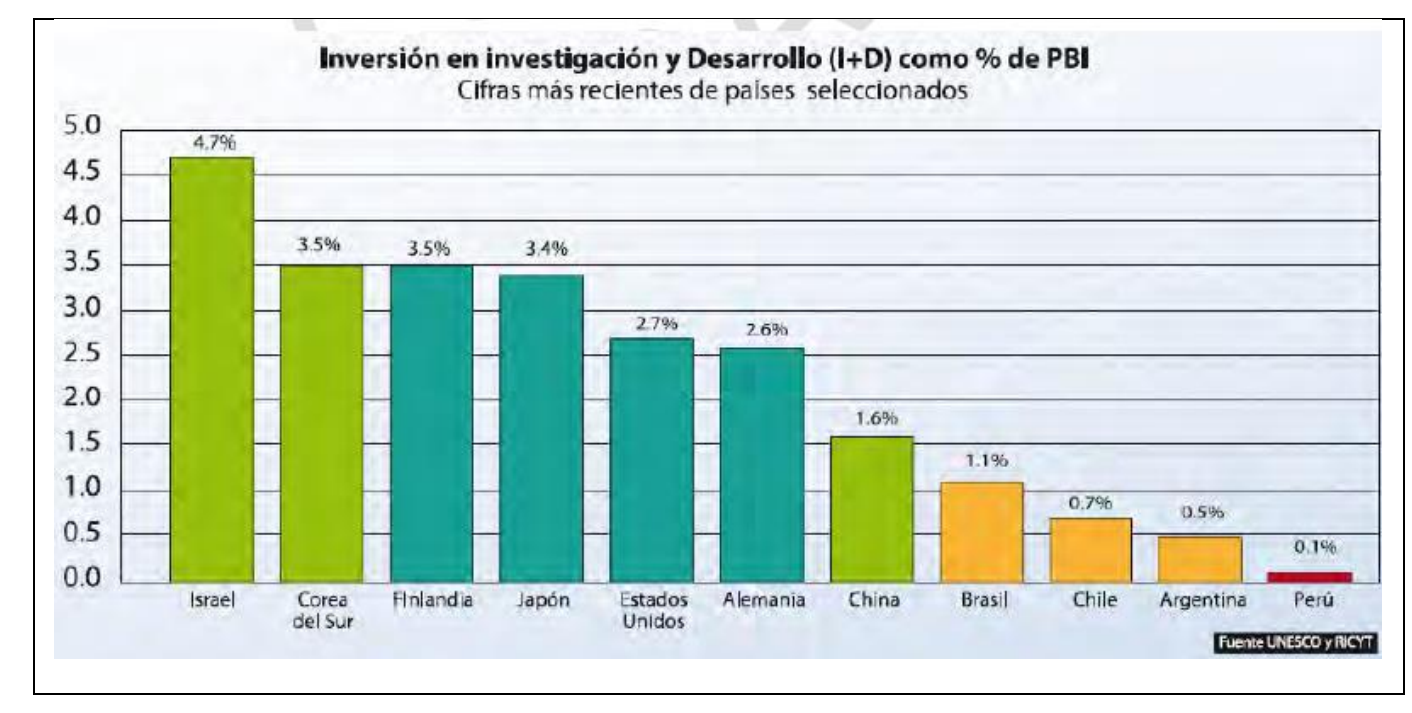

La baja inversión económica en el rubro de investigación y desarrollo científico y tecnológico es lo que trae como consecuencia, el privar de beneficios económicos a los pocos investigadores que existen, lo que perjudica la producción de conocimiento científico. Por ejemplo, las publicaciones de los artículos científicos en las revistas indexadas no son remuneradas, ni se recompensa la labor investigativa de los docentes universitarios, que tienen que sacrificar su tiempo libre y sus propios medios para la realización de esta tarea.

Este tipo de situación en nuestro medio, hace muy poco atractivo y alentador el trabajo intelectual y científico para los jóvenes. Además, la juventud de hoy está muy influenciada por los cambios y el rápido acceso a la obtención de bienes materiales que satisfacen sus expectativas presentes y, no están dispuestos a sacrificar su tiempo emprendiendo la larga preparación académica, constante disciplina, dedicación del tiempo y de los medios, la búsqueda del apoyo material y moral, es decir: todo lo que exige una verdadera investigación. Al déficit de recursos humanos, debemos agregar el ingrediente logístico de la investigación, la escasez de inversión en la infraestructura investigativa: laboratorios, materiales e instrumentos técnicos, etc., vitales para el desarrollo científico y tecnológico.

Sin embargo, aun suponiendo que a nivel político se decide favorablemente incrementar los recursos materiales, esto no podrá solucionar el problema del avance de la investigación sin la potenciación de la calidad formativa de los investigadores en las universidades.

Actualmente, según (Bermúdez García, 2014), la universidad en el Perú no es concebida como una institución de gran nivel académico productora de ciencia y tecnología, mucho menos de innovación. Es considerada exclusivamente como formadora de profesionales. Por esta razón, la gran mayoría de las universidades del Perú no producen ciencia y tecnología en los niveles de calidad que en los tiempos actuales demanda. En efecto, el país ha adoptado a larga escala las políticas educativas basadas en competencias que promueven saberes prácticos, reproductivos y aplicativos destinados a capacitar a la mano de obra técnicamente calificada, más que todo para la producción, desinteresándose —casi por completo—, de la formación de investigadores competentes en conocimiento científico con saberes sistemáticos bien ahondados que garanticen la generación, transferencia y adecuación de la ciencia y la tecnología a las necesidades sustantivas del país.

El enfoque por competencias transforma sustancialmente la finalidad de la educación superior que, en lugar de ser el centro de la transmisión y generación del saber sistemático basado en la investigación, es más orientada a la eficiencia y eficacia de la gestión y a la pertinencia de los perfiles académicos y profesionales que son requeridos en el mundo productivo del momento (Cuba Esquivel, 2016). Con esta prioridad la investigación que de principio demanda una fuerte base del saber sistemático, no encuentra su sustento estructural en el ambiente académico.

A los factores antes indicados, que desfavorecen la investigación en Perú, nosotros queremos añadir uno más: que a nuestro parecer repercute en el estado del desarrollo científico y tecnológico nacional y que es la deficiencia en la formación de las cualidades y capacidades investigativas en los estudiantes, debido a la ausencia en los planes de estudio de las instituciones educativas de disciplinas como: filosofía, lógica y epistemología que, por antonomasia, son propedéuticas a cualquier tipo de proceso investigativo.

Es utópico formar un investigador científico con hábitos cognitivos y analíticos pertinentes, para generar nuevos conocimientos técnicos y científicos sin que él tenga la actitud y aptitud de cuestionar, dudar y mantener la curiosidad que plasma la filosofía; sin capacidad de razonamiento, que educa la lógica y, sin comprensión de los principios científicos válidos que brinda la epistemología. Sin embargo, la 
realidad demuestra que las tres disciplinas aludidas son prácticamente suprimidas de la educación universitaria, como consta en la siguiente tabla:

\begin{tabular}{|c|c|c|c|c|c|c|}
\hline UNIVERSIDAD & FACULTAD & FILOSOFÍA & LÓGICA & $\begin{array}{l}\text { EPISTEMO } \\
\text { LOGÍA } \\
\end{array}$ & PERIODO & REFERENCIA \\
\hline \multirow{3}{*}{$\begin{array}{l}\text { UNIVERSIDAD } \\
\text { NACIONAL MAYOR } \\
\text { DE SAN MARCOS }\end{array}$} & $\begin{array}{l}\text { Escuela } \\
\text { Profesional de } \\
\text { Educación } \\
\end{array}$ & II sem: & II sem: & V. Sem: & $\begin{array}{l}\text { Plan de estudios del } \\
2013\end{array}$ & $\begin{array}{l}\text { http://educacion.unmsm.edu.pe/ } \\
\text { pdf/plan_estudio.pdf }\end{array}$ \\
\hline & $\begin{array}{l}\text { Escuela } \\
\text { Profesional de } \\
\text { Ingeniería } \\
\text { Industrial }\end{array}$ & $\mathrm{x}$ & $\mathrm{x}$ & $\mathrm{x}$ & $\begin{array}{l}\text { Plan de estudios del } \\
2013\end{array}$ & $\begin{array}{l}\text { Aprobado por Resolución } \\
\text { Rectoral } \mathrm{N}^{\circ} 04163-\mathrm{R}-13 \mathrm{del} \\
16.09 .2013\end{array}$ \\
\hline & $\begin{array}{l}\text { Facultad de } \\
\text { Psicología }\end{array}$ & $\bar{X}$ & $\mathrm{x}$ & $\mathrm{x}$ & $\begin{array}{l}\text { Plan de estudios del } \\
2014 .\end{array}$ & $\begin{array}{l}\text { http://psicologia.unmsm.edu.pe/i } \\
\text { ndex.php/psicologia/plan-de- } \\
\text { estudios-2014 }\end{array}$ \\
\hline \multirow{2}{*}{$\begin{array}{l}\text { PONTIFICA } \\
\text { UNIVERSIDAD } \\
\text { CATÓLICA DEL } \\
\text { PERÚ }\end{array}$} & $\begin{array}{l}\text { Escuela } \\
\text { Profesional de } \\
\text { Educación }\end{array}$ & I Semestre & $\mathrm{x}$ & $\mathrm{x}$ & $\begin{array}{l}\text { Plan de estudios del } \\
2011-2017\end{array}$ & $\begin{array}{l}\text { http://facultad.pucp.edu.pe/educ } \\
\text { acion/carreras-y-planes- } \\
\text { especiales/educacion- } \\
\text { inicial/plan-de-estudios/ }\end{array}$ \\
\hline & $\begin{array}{l}\text { Escuela } \\
\text { Profesional de } \\
\text { Ingeniería Civil }\end{array}$ & $\mathrm{X}$ & $\mathrm{x}$ & $\mathrm{x}$ & $\begin{array}{l}\text { Plan de estudios del } \\
\text { 2016-II }\end{array}$ & $\begin{array}{l}\text { http://files.pucp.edu.pe/facultad/ } \\
\text { ingenieria/wp- } \\
\text { content/uploads/2015/10/251529 } \\
\text { 17/PE-Ing--Civil-2016-2.pdf }\end{array}$ \\
\hline $\begin{array}{l}\text { UNIVERSIDAD } \\
\text { CAYETANO } \\
\text { HEREDIA } \\
\end{array}$ & $\begin{array}{l}\text { Facultad de } \\
\text { Medicina }\end{array}$ & I semestre & $\mathrm{x}$ & $\bar{x}$ & $\begin{array}{l}\text { Plan de estudios } \\
\text { vigente en el } 2016\end{array}$ & $\begin{array}{l}\text { http://www.upch.edu.pe/famed/i } \\
\text { mages/plan/PLAN_DE_ESTUD } \\
\text { IOS_2016_CARRERA_DE_ME } \\
\text { DICINA.pdf }\end{array}$ \\
\hline $\begin{array}{l}\text { UNIVERSIDAD } \\
\text { PERUANA DE } \\
\text { CIENCIAS } \\
\text { APLICADAS }\end{array}$ & $\begin{array}{l}\text { Facultad de } \\
\text { Ingenierías: } \\
\text { Ingeniería de } \\
\text { Sistemas de la } \\
\text { Información }\end{array}$ & $\bar{X}$ & $\mathrm{x}$ & $\mathrm{x}$ & $\begin{array}{l}\text { Plan de estudios del } \\
2016\end{array}$ & $\begin{array}{l}\text { http://pregrado.upc.edu.pe/sites/ } \\
\text { default/files/upc/pregrado/pagin } \\
\text { as_carreras/es/ingenieria_de_sist } \\
\text { emas_de_informacion_/malla_c } \\
\text { urricular/pagina_descargas/inge } \\
\text { nieria_de_sistemas_de_la_infor } \\
\text { macion.pdf }\end{array}$ \\
\hline $\begin{array}{l}\text { UNIVERSIDAD } \\
\text { NACIONAL DE } \\
\text { EDUCACIÓN } \\
\text { ENRIQUE GUZMÁN } \\
\text { Y VALLE. }\end{array}$ & $\begin{array}{l}\text { Facultad de } \\
\text { Pedagogía y } \\
\text { Cultura Física }\end{array}$ & VI semestre & $\mathrm{x}$ & $\mathrm{x}$ & $\begin{array}{l}\text { Plan de estudios del } \\
2016\end{array}$ & $\begin{array}{l}\text { http://www.admision.une.edu.pe } \\
\text { /admision/carreras.php?mid=7\& } \\
\text { area }=36\end{array}$ \\
\hline \multirow[t]{2}{*}{$\begin{array}{l}\text { UNIVERSIDAD } \\
\text { NACIONAL SAN } \\
\text { ANTONIO ABAD DE } \\
\text { CUSCO }\end{array}$} & $\begin{array}{l}\text { Facultad de } \\
\text { Educación y } \\
\text { Ciencias de la } \\
\text { Comu } \\
\text { nicación. }\end{array}$ & $\begin{array}{l}\text { III Sem: } \\
\text { Seminario de } \\
\text { Filosofía }\end{array}$ & $\mathrm{X}$ & $\mathrm{X}$ & $\begin{array}{l}\text { Plan de estudios del } \\
2005\end{array}$ & $\begin{array}{l}\text { http://www.unsaac.edu.pe/acade } \\
\text { mico/curriculasep/P28Educacio } \\
\text { nSecundariaCusco.pdf }\end{array}$ \\
\hline & $\begin{array}{l}\text { Especialidad: } \\
\text { Ciencias Sociales. }\end{array}$ & $\begin{array}{l}\text { III Sem: } \\
\text { Seminario de } \\
\text { Filosofía. } \\
\text { FPE } \\
\text { IV Seme: } \\
\text { Filosofía } \\
\text { Antigua y } \\
\text { Medieval } \\
\text { V sem: } \\
\text { Filosofía } \\
\text { Moderna y } \\
\text { Contemporánea: } \\
\end{array}$ & $\begin{array}{l}\text { FPE: } \\
\text { II. sem. } \\
\text { Lógica Formal } \\
\text { y Dialéctica }\end{array}$ & $\begin{array}{l}\text { FPE. } \\
\text { VII Sem: } \\
\text { Teoría del } \\
\text { Conocimiento } \\
\text { y } \\
\text { Epistemología }\end{array}$ & $\begin{array}{l}\text { Plan de estudios del } \\
2005\end{array}$ & $\begin{array}{l}\text { http://www.unsaac.edu.pe/acade } \\
\text { mico/curriculasep/P28Educacio } \\
\text { nSecundariaCusco.pdf }\end{array}$ \\
\hline $\begin{array}{l}\text { UNIVERSIDAD } \\
\text { ANDINA DEL CUSCO }\end{array}$ & $\begin{array}{l}\text { Facultad de } \\
\text { Ciencias y } \\
\text { Humanidades }\end{array}$ & $\begin{array}{l}\text { II sem: } \\
\text { Pensamiento } \\
\text { Filosófico Ético } \\
\text { y Ciudadanía. }\end{array}$ & $\mathrm{x}$ & $\mathrm{x}$ & $\begin{array}{l}\text { Plan Curricular del } \\
2016\end{array}$ & $\begin{array}{l}\text { http://www.uandina.edu.pe/inde } \\
\text { x.php/malla-curricular- } \\
\text { educacion/ }\end{array}$ \\
\hline
\end{tabular}


A modo de ejemplo, presentamos la siguiente tabla con los datos del reporte de Unesco, que evidencia la enseñanza de los cursos de filosofía en el mundo, en el que consta con color rosado los países donde la enseñanza de la filosofía se encuentra en el estado de fortalecimiento, color violeta donde el estado de fortalecimiento es acompañado con áreas de declino, color celeste indica evidente declino, y color crema indica statu quo (estado de las cosas en un determinado momento), sea positivo, sea negativo, el Perú se encuentra en estado de solipsismo filosófico.

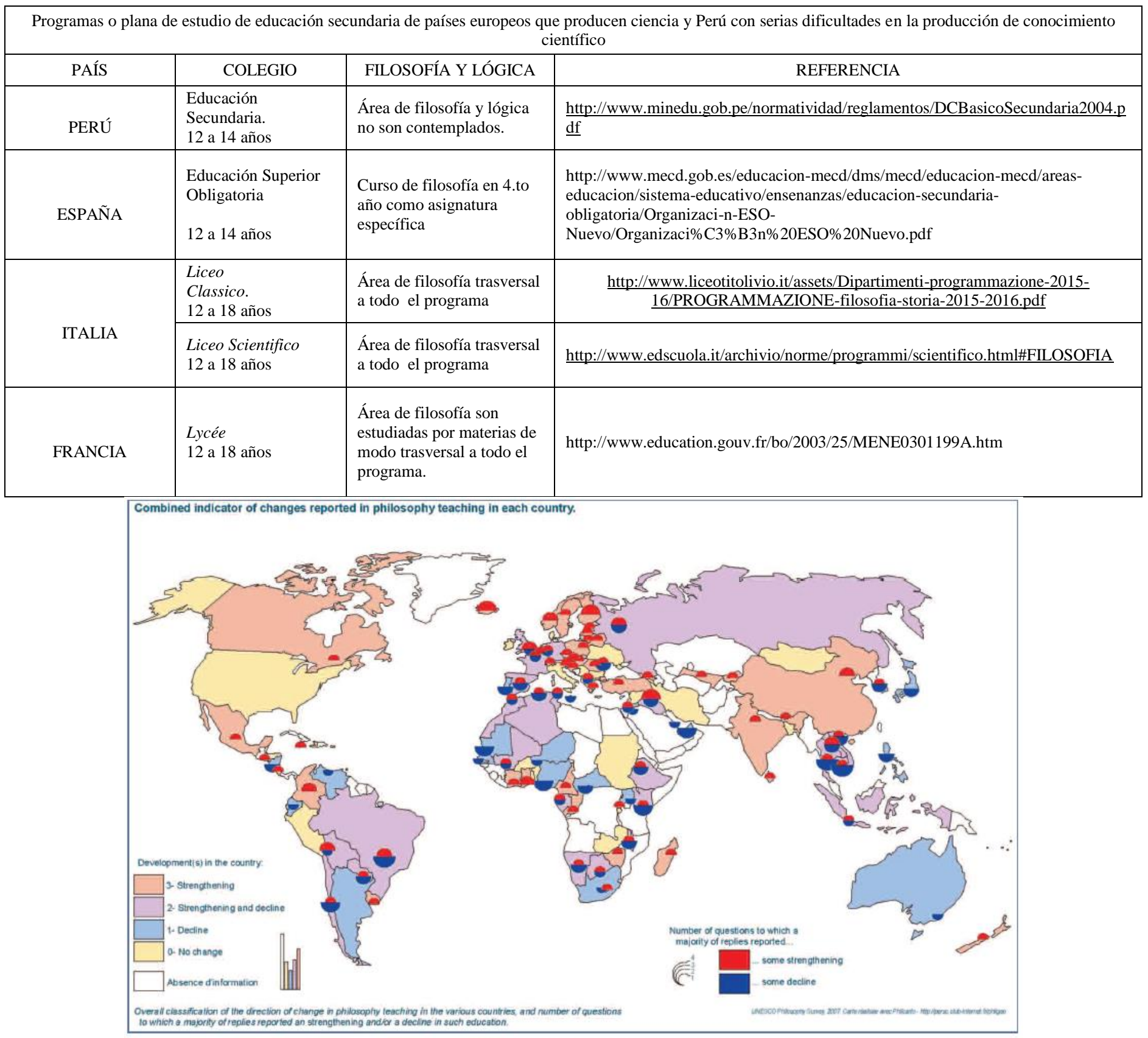

Philosophy, a School of Freedom, Paris, UNESCO Publishing, 2007, p. 221 
Según los datos de la Unesco, la filosofía es una de las disciplinas claves en los países muy avanzados en la producción científica. Esta disciplina es obligatoria en la escuela segundaria. Actualmente Unesco ha elaborado un programa de estudio de la filosofía a nivel prescolar y de la escuela primaria, intitulado Philosophy for Children (P4C) (Montclair State, 2014), y aplicada en las países europeos como proyecto piloto.

En la siguiente tabla se presenta algunos ejemplos de la presencia del curso de filosofía en los planes de estudios de algunos países europeos en comparación con el Perú:

Como constan los datos en la tabla, en los países occidentales la filosofía se estudia principalmente al nivel de educación secundaria superior (desde 14-18 años), de modo profundizado durante algunos años, ya que esta disciplina se considera propedéutica para el ingreso a la universidad.

Mientras en las universidades peruanas, para completar vacíos que deja la escuela secundaria, en los primeros dos semestres del plan de estudio prevén los cursos de formación general y, entre ellos, incluyen también filosofía, pero a modo de introducción como parte de la cultura general y no como asignatura propedéutica para investigación.

Vista la situación, queremos proponer una reflexión para demostrar que las disciplinas como la filosofía, lógica y epistemología tienen una importancia imprescindible y estratégica para la formación de las capacidades analíticas e investigativas de los estudiantes y, que la omisión de dichos cursos es un factor de la deficiencia de la investigación en el Perú.

\section{Formación filosófica del investigador}

La filosofía es transcendental para la formación de la disposición y actitud investigativa en los educandos y sus capacidades intelectuales, de la misma manera para la adquisición de una visión global e integral del saber, ya que ella es una disciplina que no solo brinda los conocimientos, sino también educa a las personas en el pensar, reflexionar, dudar, buscar nuevos conocimientos. Por tal razón, se debe tener en consideración dos aspectos formativos: subjetivo y objetivo que ofrece la filosofía, indispensables en el campo de la investigación.

La filosofía es algo que el hombre hace y siempre lo ha hecho, por ser racional, ya que racionalidad es una cualidad innata, es decir, la necesidad natural que induce al hombre a admirar, dudar, buscar los por qué de las cosas, tomar conciencia de su propio límite existencial y sufrimiento.

Según Platón y Aristóteles la admiración es el principio u origen de la filosofía. Esto se debe porque el hombre cuenta con la capacidad de asombro y de extrañeza que siente ante la realidad que lo rodea, que aviva en él la necesidad insaciable, el deseo de saber (Salazar Bondy, 1968, pág. 17)

Asimismo, según (Descartes, 2010, pág. 16), el hombre $d u d a$ ante la realidad que conoce y percibe en una situación de incertidumbre, la cual provoca una serie de preguntas que llevan a la reflexión que incita a efectuar un examen crítico de toda situación que percibe y del conocimiento que posee. Esta actitud, constituye el cimiento a partir del cual logramos conquistar el terreno de la certeza. El ser humano por naturaleza, no tolera la incerteza. Por eso, el estudio de la filosofía orienta esta inquietud natural a la búsqueda de las respuestas a las cuestiones planteadas por la vida, la sociedad y, finalmente, por la ciencia.

En efecto - el hombre siendo por naturaleza sujeto del acto de filosofar-, cuando estudia la filosofía y ejerce la actividad filosófica desarrolla sus cualidades naturales como curiosidad, reflexión, meditación, contemplación, creatividad; que no son sino más que la capacidad de buscar e investigar. El estudio de la filosofía activa y potencia con pertinencia didáctica y metodológica, todas estas capacidades naturales que se perfeccionan mediante actos sucesivos concretos, formando el hábito del investigador en la persona.

El segundo aspecto es el objeto de estudio de la filosofía, que consiste en la búsqueda «de los principios y de las causas primeras del ente en cuanto ente». (Aristóteles, 1994, pág. 15). Es decir, induce al hombre a indagar las esencias de las cosas, las razones últimas, es, más bien, una tendencia al descubrimiento, es una conquista de la razón humana. Como su objeto de estudio exige ir más allá de los fenómenos y penetrar en las profundidades del objeto que busca, esta actitud no solo incita a profundizar y fundamentar los conocimientos adquiridos sino también, busca comprender la totalidad de la existencia. Con esta actitud, la filosofía trasciende todo lo particular y tiende a abarcar lo universal, ya que busca los principios, causas de la existencia; pretendiendo comprender la totalidad. Esta actitud pretenciosa de la actividad filosófica, induce al hombre no solo a desarrollar la actitud investigativa que entraña, sino también forma y perfecciona sus facultades, instiga a fundamentar sus conocimientos, impulsa a generar el conocimiento científico, ordena y dirige las acciones que acomete, incita a buscar e investigar el sentido de la existencia; en sustancia: empodera al hombre del hábito de la ciencia, de una actitud constante de investigar hasta llegar a las últimas consecuencias.

En otras palabras, por un lado el estudio de la filosofía desarrolla las capacidades racionales naturales del hombre y las predispone al acto de investigar formando los hábitos intelectuales, actitud crítica y analítica. Por otro lado, el mismo objeto de estudio de la filosofía educa a la persona a tender a una visión universal y sistémica del mundo con una perspectiva mental abierta, rica de las asociaciones e interrelaciones indispensables para un verdadero investigador. 
Ninguna otra disciplina como la filosofía ofrece tantas bondades pertinentes para la formación del investigador. Excluyendo la filosofía de los planes curriculares, las universidades se privan del fundamento mismo de la actividad investigativa, permaneciendo en el statu quo de la situación actual de la investigación.

\section{Formación lógica del investigador}

Así como los actos naturales de las manos del músico pueden sujetarse a ciertas reglas o técnicas dando origen a los sonidos musicales, así también los actos naturales de la razón pueden supeditarse a ciertas reglas y a la dirección expresa de la propia razón, en virtud de las cuales el intelecto realiza la investigación y alcanza la posición de la verdad con cierta facilidad y seguridad.

Casi todos, tenemos experiencia de nuestra capacidad lógica cuando nos comunicamos cada día con cierta facilidad y espontaneidad, esto nos sucede porque la lógica es inherente a la naturaleza humana, por eso, se habla de la lógica natural, la cual es una aptitud natural innata para razonar que todos los hombres poseen en menor o mayor grado.

Por tal razón, la lógica se revela como una aptitud humana intelectual, una capacidad que reside en la inteligencia del hombre dando orden, congruencia interna a los actos de pensar, es decir: haciendo coherente nuestro modo de pensar.

La lógica, como disciplina científica, se desprende de la lógica natural y estudia las leyes y reglas del pensamiento, es decir, reflexiona acerca del procedimiento natural de la razón de la cual deduce una serie de reglas que ordenan y sistematizan las inferencias (Lehmann, 1965, pág. 23). De este modo, la lógica científica es una técnica sistemáticamente desarrollada y complementaria del uso de la razón humana. Por lo tanto, la lógica es un auxilio poderoso y necesario en la dirección de las operaciones que efectúa la inteligencia por medio de los procedimientos de análisis para establecer la validez de las inferencias deducidas o conocimientos generados.

Además, la lógica está relacionada con todas las ciencias, porque constituye el aspecto formal de todas ellas, es decir, desde el punto de vista formal todas las ciencias están integradas por conceptos, juicios y razonamientos, los cuales son la parte elemental del objeto de la lógica. Por otro lado, la lógica proporciona a todas las ciencias la teoría para las demostraciones científicas, es decir, no se puede hablar de ciencia si esta no está organizada conforme a las leyes lógicas, porque estas son las que garantizan su consistencia epistémica.

En sustancia, la lógica es columna vertebral de los conocimientos científicos, es implicada intrínsecamente en la aplicación de la metodología científica y, en general, no hay campo de la ciencia ni de la tecnología contemporánea donde la lógica no sea utilizada. Por eso, con gran acierto dicen (Cohen, Morris R. y Nagel, Ernest, S/F, pág. 2): «el método científico es persistente aplicación de la lógica como característica común a todo conocimiento razonado.»

Cada investigación para tener validez debe demostrar la coherencia interna de sus procedimientos y de sus conclusiones teóricas. Esta coherencia, a su vez, debe obedecer a las leyes y reglas del pensamiento brindados por la lógica. Un investigador que domina las normas lógicas con más competencia, formula las hipótesis, deduce las inferencias y argumenta con mayor nivel de persuasión; formula las teorías que con mayor grado de resistencia a la confutación.

La omisión del curso de la lógica del currículo universitario, conlleva a la incapacidad de los estudiantes de plantear problemas de las tesis, formular las hipótesis, arribar a las conclusiones coherentes, tienen una dificultad en la redacción lógica del texto científico (Maridueña M., I.J. y Astudillo Q., M. A., 2016, pág. 47). Si los estudiantes se fatigan al construir de modo lógico sus trabajos ¿cómo pueden ellos emprender una actividad relevante de una investigación científica? El dominio de la lógica es fundamental, ya que es un instrumento ineludible para cualquier discurso científico. La universidad, al privar a los educandos de la enseñanza de la lógica, les priva de una herramienta de acceso a los procedimientos de investigación.

\section{Formación epistemológica del investigador}

La ciencia es un conjunto de conocimientos objetivos, rigurosos y verificables, sistemáticamente ordenados sobre una determinada parte de la realidad objetiva (Bunge, 1994, pág. 13). Siendo así, ella cuenta con un objeto de estudio, con el principio que la fundamenta, con el marco teórico y la metodología. El pensamiento científico, así como el conocimiento filosófico, necesita de la lógica para establecer su coherencia y validez del conocimiento que concibe, así también, él requiere de la epistemología para verificar y justificar la consistencia de los conocimientos científicos que las producen.

La epistemología proviene etimológicamente de la vos griega: Episteme $(\dot{\varepsilon} \pi l \sigma \tau \eta \dot{\mu \eta})=$ ciencia, y logos $=$ tratado, es decir "ciencia del saber". Pese a esto, históricamente - tanto lógica como epistemología — siempre han estado presentes en la generación del conocimiento sea filosófico como científico. Sin embargo, en el marco de la filosofía moderna ella se constituye como una disciplina que estudia el "conocimiento científico" (Morsilli, 2001). Alrededor de 1920 a 30, la epistemología se establece como disciplina autónoma por obra de los neopositivistas del «Círculo de Viena y de Berlín», quienes dieron un gran impulso atribuyéndoles el estudio crítico y lógico de los estatutos de las teorías científicas. 
Según (Lalande, 1967), la epistemología, se ocupa esencialmente del «estudio crítico de los principios, de las hipótesis y de los resultados de las diversas ciencias, destinados a determinar su origen lógico, consistencia y el alcance de su objeto». Por ende, la epistemología se constituye de notable importancia para la producción y legitimación de todos los conocimientos científicos.

Ahora bien, ¿por qué la epistemología requiere ser estudiada por el investigador en la producción del conocimiento científico? Esta pregunta revela tres valiosas alternativas: En sentido lato, esta disciplina ayuda al investigador a esclarecer las condiciones de posibilidad en que se dan los conocimientos de la realidad, ayuda a analizar y juzgar, usando criterios objetivos, la validez y alcance de las teorías científicas mediante los distintos métodos de comprobación o refutación, así como a detectar los límites e inconsistencia de los conocimientos. En sentido estrecho, ella ayuda al investigador a examinar desde diversas perspectivas la idoneidad lógica de empleo correcto del método de investigación, los procesos de verificación, sus fundamentos, la validez de sus resultados, sus estructuras formales y lingüísticas, relaciones formales entre los enunciados científicos, significados de términos; monitorea la dirección y la organización intelectual de la ciencia, ayuda a resolver problemas científicos y filosóficos, reconstruir las teorías científicas de manera axiomática, solucionar las aporías (enunciado que expresa o que contiene una inviabilidad de orden racional) internas en las teorías científicas, sirve de modelo a todas la ciencias (básica y aplicadas), etc.

Asimismo, la epistemología no solo se constituye como instrumento indispensable en la producción y legitimación de todas las ciencias naturales y humanas, sino también se convierte en una herramienta imprescindible para el investigador, porque ella proporciona bases para generar nuevos conocimientos científicos y verificar la consistencia de los mismos, comprobar críticamente los paradigmas asumidos, ver la lógica del procedimiento intelectual que desarrolla durante el proceso de investigación, etc.

La epistemología empodera al investigador, según (Matos, E., y Fuentes, H., 2016, pág. 29), de los «procedimientos intelectuales sustentados en la argumentación lógica-científica que propicia desarrollar el pensamiento teórico». Por lo tanto, el investigador con formación epistemológica cuenta con plataforma bien implementada, a partir de la cual puede reflexionar sobre el objeto, conocimiento, resultados, métodos, validez, alcance, lógica, paradigmas, límites, en fin; los criterios a partir de los que despliega el análisis epistemológico de su objeto, etc.

Una investigación científica sin referencia a los principios epistemológicos, es una investigación con poca trascendencia, priva del valor teórico universal y puede tener exclusivamente carácter contingente y aplicativo, sin capacidad de generar nuevos conocimientos y como consecuencia, infértil.

\section{Conclusión}

Las bondades de la filosofía, lógica y epistemología son indispensables para formar un investigador en la producción del conocimiento humano. La filosofía congrega las condiciones indispensables para educar la actitud del investigador, potenciando y formando sus cualidades naturales y adquiridas del hábito de investigar. La lógica empodera al investigador de cualidades para el razonamiento correcto, y la epistemología brinda los criterios lógicos, conceptuales, lingüísticos, metodológicos que legitiman un conocimiento como ciencia.

Si se desea fortalecer la investigación en el Perú, no solo se deben solucionar los problemas económicos y logísticos, sino también es necesario tomar medidas estratégicas de largo plazo con perspectiva del futuro, partiendo de la formación del cuerpo investigativo con el perfil pertinente dotado de todas las cualidades indispensables plasmadas gracias al estudio de la filosofía, lógica y epistemología.

\section{Referencias bibliográficas}

Aristóteles. (1994). Metafisica: Testo greco a fronte (2.da ed.). (C. Rossitto, Ed.) Milan, Italia: Rusconi.

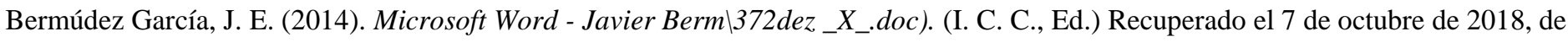
http://www.detrasdelacortina.com.pe/images/download/Investigacion\%20cientifica\%20como\%20factor\%20de\%20desarrollo _Javier\%20Bermudez\%20Garcia.pdf

Bunge, M. (1994). La ciencia, su método y su filosofía. Buenos Aires, Argentina: Siglo Veinte.

Cohen, Morris R. y Nagel, Ernest. (S/F). La lógica y el método de la ciencia. (C. V. Molina, Ed.) Obtenido de http://twiki.org/p/pub/Main/VictorMartinez/Mtodo_de_la_Ciencia-Capitulo_I.pdf

Comisión Consultiva para la Ciencia, T. e. (2012). Nueva política e institucionalidad para dinamizar la CTI Peruana. . Política de Estado, Presidencia del Consejo de Ministros, Lima. Obtenido de http://franciscosagasti.com/descargas/eventos/ff-informecomision-

Cuba Esquivel, A. (Marzo de 2016). Constructo competencia: síntesis histórico-epistemológica. Educación, XXV(48), 7 - 27. Recuperado el 6 de octubre de 2018, de http://www.scielo.org.pe/pdf/educ/v25n48/a01v25n48.pdf 
Descartes, R. (2010). Discurso del Método (6.ta ed.). (M. G. Morente, Trad.) Barcelona, España: Espasa Calpe.

Lalande, A. (1967). Vocabulario técnico y crítico de la filosofía (2.da ed.). Buenos Aires, Argentina: El Ateneo.

Lehmann, R. (1965). Introducción a la lógica. (T. d. Marías., Trad.) Buenos Aires, Argentina: Editorial Losada S. A.

Maridueña M., I.J. y Astudillo Q., M. A. (26 de abril de 2016). Causas de la baja productividad en artículos indexados de la UNEMI. Revista Científica Ciencia y Tecnología, 1(11), 42 - 51. Obtenido de file:///C:/Users/Intel/Downloads/12-30-1-PB.pdf

Matos, E., y Fuentes, H. (2016). Lo epistemológico en la lógica del proceso investigativo. Santa Fe de Bogotá, Colombia. Obtenido de https://www.ecured.cu/Bases_epistemol\%C3\%B3gicas

Montclair State, U. (2014). ¿Qué es la "Filosofía para niños"? Red Social Universitaria. Recuperado el octubre5 de 2018, de https://www.montclair.edu/cehs/academics/centers-and-institutes/iapc/what-is/

Morsilli, E. (2001). Dizionario di filosofia e scienze umane. (C. S. Editore, Ed., \& C. P. R. Infante, Trad.) Milano, Italia: libreríauniversitaria.it.

Salazar Bondy, A. (1968). Introducción a la filosofía. (R. Q. Avila, Ed.) Lima, Perú: Universo.

SINACYT - CONCYTEC. (2008). Propuesta de plan nacional de ciencia, tecnología e innovación tecnológica para el desarrollo productivo y social sostenible. Lima. Lima: CONCYTEC. Recuperado el 8 de noviembre de 2018, de http://www2.produce.gob.pe/RepositorioAPS/3/jer/TALLER_PNDA/PNCTI\%202008\%20\%E2\%80\%93\%202012.pdf

UNESCO (1996). Learning: The Treasure Within, Report to UNESCO of the International Commission on Education for the Twentyfirst Century, Paris, UNESCO Publishing. Recuperado de: http://unesdoc.unesco.org/images/0010/001095/109590eo.pdf

UNESCO, (2011) Teaching Philosophy in Europe and North America. Paris, Francia: United Nations Educational, Scientific and Cultural Organization. Recuperado de: http://unesdoc.unesco.org/images/0021/002140/214089e.pdf
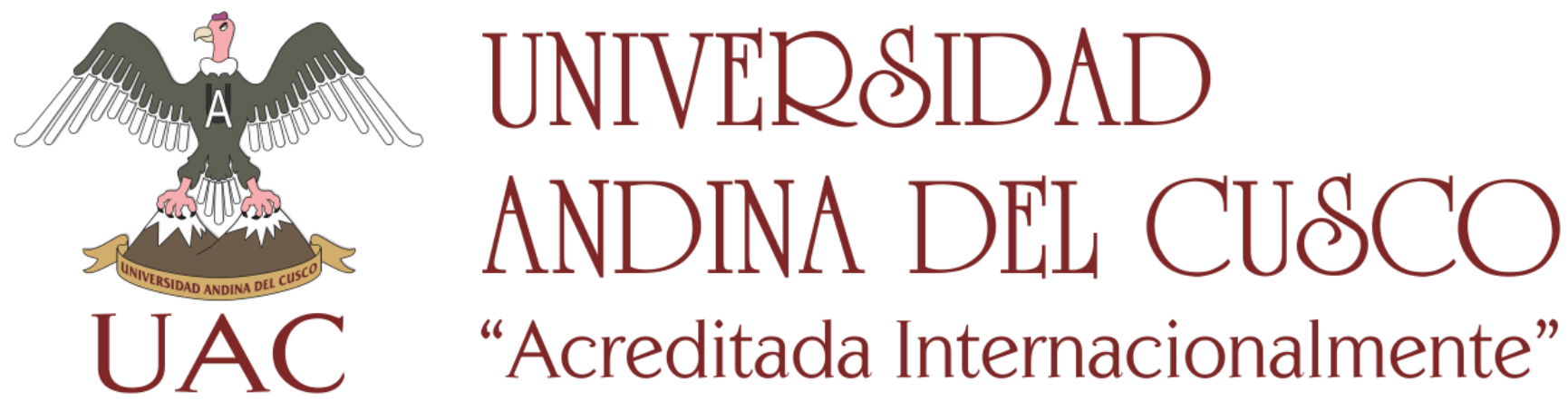

Rev. Yachay volumen (7) Número (1), enero-diciembre 2018 